\title{
Flow Cytometry and Effusions in Lymphoproliferative Processes and Other Hematologic Neoplasias
}

\author{
Beata Bode-Lesniewska \\ Institute of Surgical Pathology, University Hospital Zurich, Zurich, Switzerland
}

\section{Key Words}

Flow cytometry · Effusions · Hematologic neoplasia

\begin{abstract}
Cytopathologists are regularly confronted with lymphocyte-rich effusions, and the definite decision of whether the lymphocytosis is of a purely reactive nature or a presentation of an indolent lymphoma may be an extremely difficult one based on microscopy alone. Flow cytometry (FC) offers many advantages in terms of its application in body cavity fluids, and it has proven to be very useful both in the setting of a known disease and for new lymphoma diagnoses. In this paper, the studies published in recent years dealing with the applications of FC in body cavity effusions in the context of hematologic neoplasia are reviewed, stressing the integrative diagnostic approach. The incorporation of microscopical, immunophenotypical, and molecular findings from examinations of the cellular content of effusions and the interpretation of results in relation to the current WHO classification of hematolymphoid malignancies give cytopathologists new perspectives on advanced and clinically highly relevant diagnostics.
\end{abstract}

(c) 2016 S. Karger AG, Basel

\section{KARGER}

(c) 2016 S. Karger AG, Basel

0001-5547/16/0604-0354\$39.50/0

E-Mail karger@karger.com

www.karger.com/acy

\section{Introduction}

The occurrence of body cavity effusions is always a symptom of a considerable pathologic process. The etiology of effusions comprises a wide spectrum of diseases ranging from nonneoplastic ones, such as posttraumatic, inflammatory (infection or connective tissue diseases), circulatory (congestive heart failure and central lymphatic obstruction), and kidney or liver dysfunction disorders, to manifestations of any kind of malignancy, either spreading from other organs (lungs, breasts, ovaries, and pancreas, etc.) or being primary [mesotheliomas and primary effusion lymphomas (PEL)]. Malignant pleural effusions are common in patients with hematologic malignancies and they are reported to occur - depending on the specific entity - in up to $48 \%$ of such patients [1-3], while involvement of the peritoneal and pericardial cavities seems to be less common. Up to $10 \%$ of malignant pleural effusions with a positive cytologic examination are due to non-Hodgkin lymphoma (NHL). In the vast majority of cases, serous effusions develop during the course of the disease, but rarely they may be the first presentation of a hematologic malignancy. The pathogenesis of effusions in lymphomas and hematologic neoplasias is 
considered to be multifactorial. The most common cause is infection due to an impaired immune system during the course of the disease or its treatment. Impaired lymphatic drainage as a result of mediastinal lymph node enlargement, thoracic duct obstruction, venous obstruction, increases in vascular permeability, and radiationassociated changes, as well as infiltration of the serosal membranes by the tumor, probably all contribute in the pathogenesis of effusions in this setting. The presence of cells of a hematologic malignancy in effusions is usually a sign of advanced and generalized disease. In most cases, it causes substantial morbidity and requires treatment. In the study of Gilbert et al. [1], among 91 patients with hematologic disease requiring placement of an indwelling pleural catheter, $62 \%$ had a diagnosis of a lymphoma, followed by leukemia (21\%), and multiple myeloma (13\%), with 4 patients having either amyloidosis or myelofibrosis.

While the presence of carcinoma, mesothelioma, or sarcoma cells in pleural effusions is microscopically obvious in most cases, the diagnosis of lymphoma cells in fluids - especially of the indolent lymphoma subtypes - may be challenging for several reasons. The cells of low-grade NHL usually have a deceptively bland cytologic appearance, while cells in reactive conditions may show a striking atypia. The possibility of characterizing individual cells according to their surface antigenicity by flow cytometry (FC) offers the chance to precisely subclassify the cells and sort them into separate populations. Body cavity fluids, as suspensions of well-preserved, viable cells, are well suitable for this kind of study.

Immunophenotyping by FC is a well-established diagnostic method in the hands of hematologists for the detection and subtyping of hematolymphoid malignancies in blood, lymph nodes, bone marrow, and other organs. The incorporation of this method into routine cytopathologic diagnostics appears to be less well established and varies among institutions; however, it has become more and more generally accepted in recent years, primarily in the setting of fine-needle aspirations (FNA) but also increasingly for the study of the composition of the cellular content of body fluids. The application of FC by cytopathologists is feasible and rewarding in relation to the diagnostic yield, especially when combined with the application of other ancillary techniques, such as immunohistochemical and molecular methods. As the diagnostic criteria of hematologic diseases tend to incorporate more and more immunogenic and molecular features of the tumor cells instead of purely architectonic attributes of the tissue, as defined in the current WHO classification [4, 5], the spectrum of final diagnoses in hematopathology within the reach of cytopathology widens. This review predominantly focuses on studies published in the last 10-15 years on the application of FC in body cavity effusions, in order to discuss the entities as they are defined in the current nomenclature of hematologic neoplasia $[4,5]$.

\section{Technical and Methodological Aspects}

Flow cytometric analysis of hematolymphoid processes in body fluids is a rapid, reproducible, sensitive, and quantitative method for immunophenotyping of cells, which utilizes a panel of fluorescent dye-tagged antibodies directed mostly against the cell surface markers. Demonstration of the presence or absence of light chain restriction is essential for the evaluation of B cells with the option of characterizing further lymphoma type-specific markers in cases with light chain restriction. A pathologic immunophenotype of $\mathrm{T}$ cells with a loss of antigen expression or an unusual coexpression of markers (e.g. CD7, CD4, and CD8) is indicative of T-cell neoplasia. Serous effusions are very well suited for studies using FC as they naturally consist of suspensions with viable cells with well-preserved surface antigens. Cells of many types of lymphomas and leukemias are particularly conveniently studied via FC analysis due to their abundance in samples and the size and type of the expressed antigens. Since the body effusions are almost always submitted for cytopathologic analysis in sufficient volumes of over $100 \mathrm{ml}$ (and, if not, this has to be changed/ adapted in the affected institutions), the subject of a low cell content is - in contrast to fluids with inherently low volumes and cell contents, such as central nervous system fluid - not a general problem. Counts of at least several thousands of cells can be reached easily for any combination of antibodies.

There is a wide range of fluorochrome-conjugated antibodies available for FC detected at a different wavelengths. The simultaneous detection of multicolored signals in modern instruments (up to several channels) provides the opportunity to evaluate multiple antigens at the same time, offering means to extensively characterize various cell populations. Selection of the antibody panel for individual study depends on the individual case; the routine panel consists in most cases of at least: CD45, $\kappa$ light chains, $\lambda$ light chains, CD19, CD20, CD3, CD5, $\mathrm{CD} 10$, and CD23. The panel can be extended in any specific case and it can incorporate further markers according to the patient history and/or the entity studied (fur- 


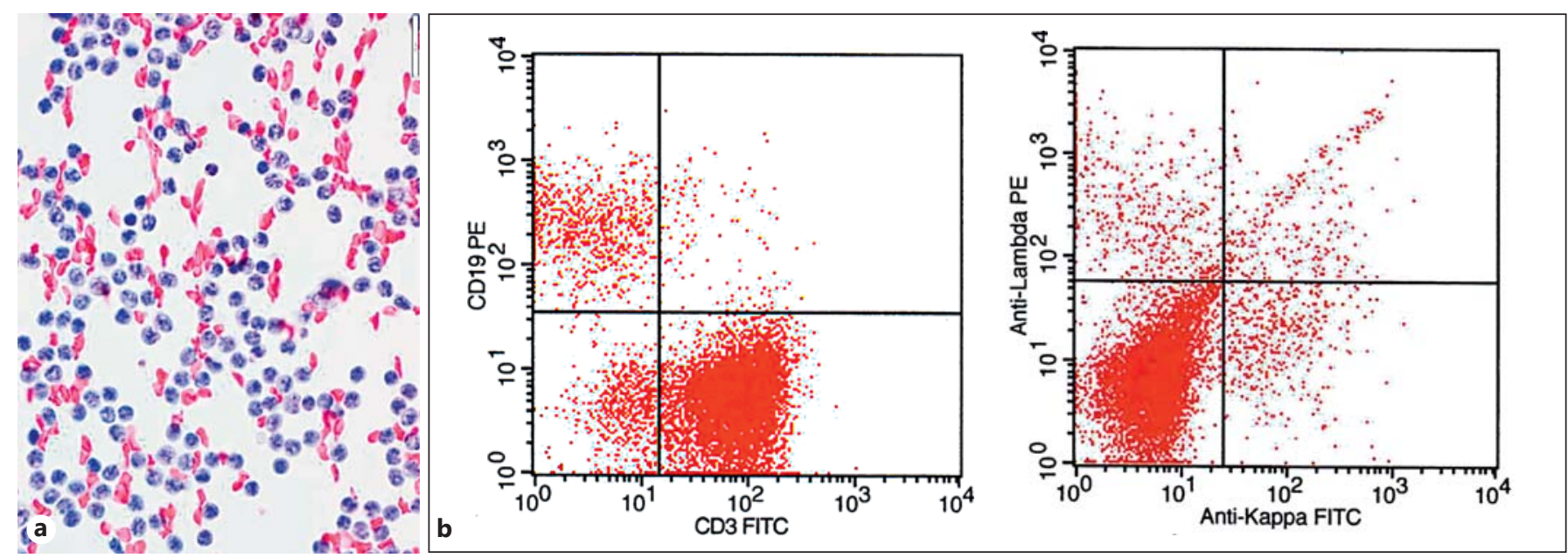

Fig. 1. Pleural fluid of an 88-year-old male patient with a history of lung tuberculosis. a Numerous small lymphocytes in the sediment of the fluid, practically without mesothelial cells. Papanicolaou. Original magnification $\times 400$. b FC demonstrating a reactive pattern with numerous CD3-positive T cells (83\%) and a small
CD19-positive B-cell population (9\%) without light chain restriction $(\kappa / \lambda$ ratio of $1: 1)$, corresponding to a reactive lymphatic population in the effusion specimen. Lymphocyte-rich pleural effusions with a low mesothelial cell count are often seen in tuberculous pleurisy. ther T-cell markers, monocytic markers, etc.). For optimal results, several thousand signals should be evaluated for every antibody combination, requiring in general at least a million viable cells in the sample; this prerequisite is easily reached in routine samples of body effusions in contrast to other specimens of lower cellularity such as central nervous system fluid or some FNA. Nonneoplastic reactive effusions usually contain numerous lymphocytes and histiocytes; the lymphocytic population consists of at least $90 \%$ CD3-positive T cells, with only a few CD19+, CD20+ polytypic B cells (fig. 1).

\section{Indication for FC of Effusions}

Correct cytologic discrimination between reactive and malignant effusions is essential for the clinical management of patients with a hematologic disease. The prognostic relevance of malignant effusions has been discussed controversially in the literature. However, recent studies suggest that its presence may be very important; in fact, patients with a positive effusion cytology, especially if present at presentation, have the worst prognosis [2]. Lymphocyte-rich body fluids are common in routine cytopathology, and the indication for auxiliary studies should be carefully made to avoid cost-ineffective studies of reactive lymphocytosis [6]. In order to ensure the judicious management of resources, it is important that not all effusion samples be automatically submitted for FC [7] but that the specimens undergo an initial microscopical review by a cytopathologist. This primary review allows the exclusion of samples containing obvious inflammation or nonhematologic malignancy from the lymphatic FC work-up. After consideration of the differential diagnosis, the proper indication should lead to selection of the antibody panel for the FC study. Since the frequency of malignant effusions is significantly higher in patients with known lymphomas than in those without a hematologic malignancy, the history of any such neoplasia, particularly of an indolent lymphoma, is the most important indication for performance of an FC analysis $[6,8,9]$. However, if the etiology of the microscopically nonneoplastic effusion remains unexplained, and especially if it is recurrent or accompanied by a striking lymphadenopathy, FC should be performed to rule out or prove the presence of an indolent lymphoma. The microscopically atypical lymphocytes should be examined by FC in order to differentiate reactive from neoplastic changes. If the fluid tap was performed in the context of an emergency clinical presentation for symptomatic relief (in adults or in children) and the sample contains an obviously malignant hematologic neoplasia, FC is indispensable since it may be rapidly indicative of lymphoma and it may lead to prompt employment of the proper treatment as shown by Bhaker et al. [10] for pediatric patients with a precursor T-lymphoblastic lymphoma. 


\section{Impact of FC on Cytologic Diagnoses}

The influence of the results of the FC on the final interpretation of the cytologic evaluation of the effusion specimen has been studied recently in several studies.

In the study of Czader and Ali [8], FC was performed primarily on effusions of patients with a known history of malignant lymphoma, and 115 cases (pleural fluid, $n=86$; peritoneal fluid, $n=20$, and pericardial fluid, $n=9$ ) were retrospectively analyzed, with a comparison of the provisional cytopathologic diagnosis and the final diagnosis, incorporating the modification by the FC result. Among the cytologically malignant cases $(n=43 ; 37 \%)$, there were 32 lymphomas, 1 case of acute lymphoblastic leukemia, 1 case of acute myelogenous leukemia, 2 cases of T-cell lymphoma/leukemia, 1 multiple myeloma, 1 Hodgkin lymphoma (HL), 4 cases of adenocarcinoma, and 1 sarcoma. In 18 cases (16\%), the provisional cytologic diagnosis was later modified by the FC results from atypi$\mathrm{cal} /$ suspicious to benign (8 cases) and from benign or atypical/suspicious to malignant [10 cases: 1 case of chronic lymphocytic leukemia (CLL), 2 cases of mantle cell lymphoma (MCL), 3 follicular lymphomas (FL), 1 large-cell lymphoma, 1 plasma cell myeloma, 1 T-cell lymphoma, and $1 \mathrm{NK}$ cell leukemia], underscoring the importance of FC as an adjunct to cytomorphology in body cavity effusions. Cesana et al. [11] analyzed the comparison of FC and cytomorphology results in 92 body cavity fluids (61 effusions and 31 bronchoalveolar lavage fluids). The overall concordance of both methods was $75 \%$ [ 56 cases (61\%) were double true negative, 11 cases (12\%) were double true positive, and 2 cases (2\%) were double false negative]. Among the discordant cases, the FC was false negative in 1 case of a cytologically apparent PEL in the pleural fluid, while the FC was positive in 8 falsely negative body fluids (5 pleural and 3 ascites) in cytomorphology only. In cytomorphologically atypical cases, a definite result was achieved by FC in 7 cases of body fluids (pleural fluid, $n=6$; ascites, $n=1$ ). The $\kappa$ value in relation to the clinical outcome was, in the group of body fluids, 0.65 for cytomorphology only and 0.97 for FC, and it reached 1.0 for the diagnostic yield of the combined method, stressing the advantages of an integrative approach. Iqbal et al. [6] retrospectively analyzed 89 body fluid specimens (pleural, $\mathrm{n}=70$; peritoneal, $\mathrm{n}=14$, and pericardial, $n=5$ ). In patients with no history of hematologic disease, the working cytopathologic diagnosis was modified from benign/atypical to malignant in only 2 (11\%) cases and from atypical/suspicious to benign in 11 (33\%) cases. In patients with a prior clinical history (38 cases), $\mathrm{FC}$ was positive in 22 cases (58\%) and negative in 16 cases $(42 \%)$. In 31 cases (35\%), the working cytopathologic diagnosis was subsequently modified by the FC results from atypical/suspicious to benign (13 cases) and from benign/atypical to malignant (18 cases). The application of FC allowed confidently for either confirmation of the diagnosis of malignancy or sure exclusion, lowering the rate of atypical or indeterminate diagnoses. The authors concluded that FC should probably be limited to cases with: (1) a documented history of hematopoietic malignancy, (2) a strong clinical suspicion of malignancy, and (3) atypical cytologic or morphologic features suspicious for a hematopoietic malignancy. In the study of $\mathrm{Yu}$ et al. [12], a 4-year retrospective analysis demonstrated the application of FC in altogether $2.7 \%$ of 6,925 body fluids (3.4\% of 3,220 body cavity effusions and $1.9 \%$ of 3,705 cerebrospinal fluid specimens). In the category of body cavity effusions, FC was applied most often in pleural effusions (4.5\%) and less often in pericardial (1.9\%) or peritoneal fluids (1.1\%). Among the effusion cases with FC studies, there were positive or atypical results in 30$67 \%$ of cases, depending on the fluid origin. The cases with atypical FC findings or atypical morphologic findings with normal FC were studied further with auxiliary methods (immunocytochemistry and molecular genetics), which led to a final definitive diagnosis in a substantial number of cases. Seven of 39 positive cases on FC pleural effusion had an as yet not diagnosed lymphoma [T-cell lymphomas, $\mathrm{n}=2$; PEL, $\mathrm{n}=1$; MCL, $\mathrm{n}=1$; marginal-zone lymphoma (MZL), $\mathrm{n}=1$; diffuse large $\mathrm{B}$-cell lymphoma (DLBCL), $\mathrm{n}=1$, and plasma cell neoplasm, $\mathrm{n}=1$ ]. Among the negative cases, 6 showed atypical morphologic features and were studied further, similarly to the cases with atypical, but nondiagnostic, findings on FC, yielding a final definitive diagnosis in an additional 29 and $17 \%$ of cases, respectively. The study of Tong et al. [9] describes an algorithmic approach, including the application of FC and other auxiliary studies [immunohistochemistry, polymerase chain reaction for B- and T-cell clonality, human herpes virus type 8 (HHV8) status, and EBER], to the diagnosis of lymphoproliferative disease in effusion samples based on 10 years of experience. A diagnosis of a lymphoproliferative disease was made in 133 pleural effusions, 30 ascites, and 5 pericardial effusions. In $77 \%$ (122 of 168) of cases a lymphoma diagnosis could be definitely made on cytology, and in $23 \%$ of cases a suspicion of lymphoma was rendered. In $38.2 \%$ of cases, the diagnosis of lymphoproliferative disease was new, while in the remaining cases the manifestation of a known disease was confirmed. The application of FC was successful 


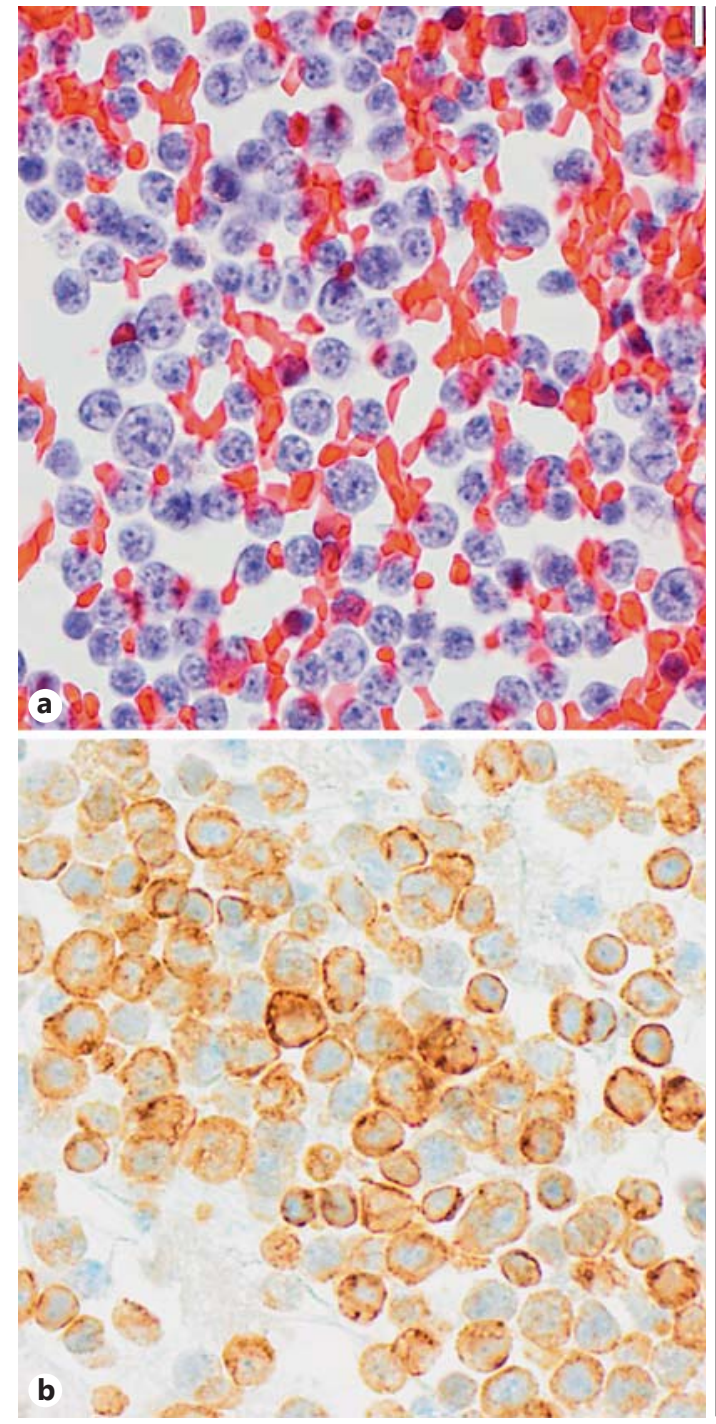

Fig. 2. Pleural effusion of an 83-year-old female patient with a history of NHL. a, b Monomorphic middle-sized lymphocytes and a few blasts in the sediment smear (Papanicolaou, original magnification $\times 400)(\mathbf{a})$ with immunocytochemical expression of the bcl2
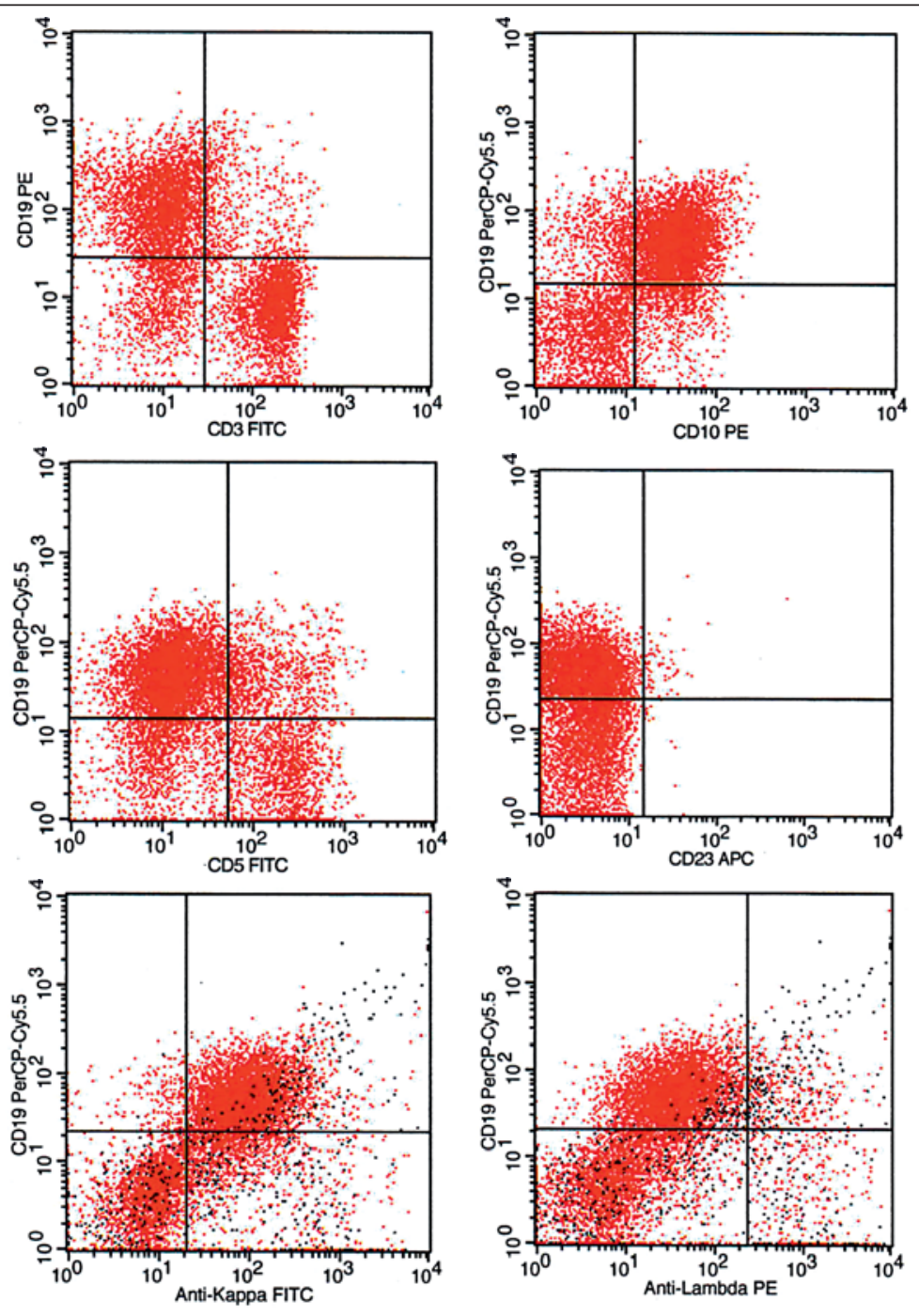

antigen (b). c FC demonstrating a large CD19-positive B-cell population with $\kappa$ chain restriction and coexpression of CD10, corresponding to the diagnosis of FL. in $74.4 \%$ of the studied cases (64 of 86), leading in most cases to a final diagnosis and covering the whole spectrum of lymphoma types: generic diagnoses in 82 patients [large-cell lymphomas, NHL not otherwise specified, small B-cell NHL $(\mathrm{n}=14)$, and T-cell lymphomas, large cell type $(n=2)$ ] or specific subtype diagnoses in 47 patients [CLL/small lymphocytic lymphoma (SLL), FL, MCL, Burkitt lymphoma (BL), PEL $(\mathrm{n}=4)$, T-ALL ( $=$ 4), posttransplant lymphoproliferative disease $(n=3)$, peripheral T-cell lymphoma $(\mathrm{n}=2)$, NOS, and 1 myeloma $(\mathrm{n}=1)]$. Multiple ancillary tests were performed with a high success rate as part of the multiparametric approach to lymphoma subtyping, similarly to other studies [13, 14].

Lymphoma/leukemia was reported to be the most frequent cause of malignant effusions in children $[3,10,15]$. In a study of children younger than 18 years with 226 cases of pleural, peritoneal, and pericardial effusions, 88 (39\%) patients were positive or suspicious of malignancy, and among these lymphoma/leukemia was found to be the most frequent cause (52\%) of malignant effusions. The role of FC immunophenotyping as a rapid diagnostic 
tool for pediatric malignancy in serous cavity effusions was studied by Shen et al. [16]. Among 41 of 103 (40\%) patients with malignancies, 36 were diagnosed by FC (lymphoma, $\mathrm{n}=25$; acute myeloid leukemia, $\mathrm{n}=2$; neuroblastoma, $\mathrm{n}=8$, and retinoblastoma, $\mathrm{n}=1$ ), and $\mathrm{FC}$ proved to be especially useful in the category of NHL.

\section{B-Cell NHL}

\section{Chronic Lymphocytic Leukemia/Small Lymphocytic} Lymphoma

CLL/SLL is a common form of an indolent hematologic malignancy that affects the older population and involves predominantly either bone marrow with blood lymphocytosis (CLL) or lymph nodes (SLL). For CLL/ SLL in autopsy series, pleural involvement has ranged from 3 to $16 \%$ [17]. The appearance of malignant cells of this lymphoma type in body cavity effusions is common, and it is diagnosed mostly several years after the first diagnosis of the tumor $[18,19]$. It can easily be proven via FC by demonstration of a light chain-restricted CD19+, $\mathrm{CD} 20+\mathrm{B}$-cell population coexpressing CD5 and CD23 but lacking CD10. Since the frequency of CLL/SLL is higher in the older population, the coexistence of other diseases with the indolent lymphoma will become more and more frequent in the future as life expectancy increases. Detection of a small fraction of CLL cells in effusions in patients with stable disease should not divert from the search for other, clinically potentially more relevant causes of body cavity fluid accumulation, such as congestive heart failure of renal insufficiency. Occasionally, a second malignancy may be observed in the background of CLL $[20,21]$, and this phenomenon may be even more common in the future as cancer turns from a uniformly fatal disease into a chronic disease under the modern management regimens. It has been shown that CLL patients are at risk for the development of further nonlymphoid malignancies, such as melanomas, carcinomas, or sarcomas, with an estimated $2.5 \%$ of CLL patients at risk for the development of lung carcinoma. With cytologic methods, including FC, both tumors, i.e. CLL and carcinomas, may be confidently diagnosed at the same time, as described by Vrettos et al. [21]. Large-cell lymphoma via Richter's transformation may also occur in 5-10\% of CLL cases.

\section{Follicular Lymphoma}

FL (fig. 2) is one of the most common lymphoma types, and it accounts for approximately $35 \%$ of all NHL cases.
It is characterized by the $\mathrm{t}(14 ; 18)(\mathrm{q} 32 ; \mathrm{q} 21)$ translocation, causing overexpression of the $b c l 2$ gene. Depending on the differentiation, it is either an indolent or an aggressive disease and may involve body cavities. FL is usually diagnosed histologically via demonstration of the typical nodular growth pattern architecture and immunophenotype. Malignant effusions as a first or only presentation of FL are an exception [22], but they belong to the differential diagnosis of an unexplained lymphocytic effusion. Demonstration of a CD19+, CD20+ cell population with a light chain restriction and CD10 positivity, complemented by demonstration of bcl 2 and bcl6 expression as well as rearrangement of the $b c l 2$ gene by fluorescence in situ hybridization (FISH), may reliably allow the diagnosis of FL in body fluids.

\section{Mantle Cell Lymphoma}

MCL (fig. 3) is a mature B-cell lymphoma characterized by the translocation $\mathrm{t}(11 ; 14)(\mathrm{q} 13 ; \mathrm{q} 32)$ involving the $b c l 1$ gene. It has a distinct long-term poor prognosis. The main involved organs are lymph nodes and the spleen, with rare extranodal presentations. It may occasionally be demonstrated in effusions, which may also occur as the first manifestation of the disease $[14,23,24]$. The typical immunophenotype of MCL including light chain restriction, i.e. CD19+, CD20+, CD5+, CD10-, CD23-, CD3-, can elegantly be demonstrated via FC and supplemented by a FISH demonstration of the typical gene rearrangement $[14,24]$.

\section{Marginal-Zone Lymphoma}

MZL is a rare type of indolent B-cell NHL. MZL is only rarely found in effusion fluids. A unique case of MZL accompanied by an extensive emperipolesis was recently described by Meykler et al. [25]. The pleural effusion was notably the first tumor manifestation, and tumor cells were identified by the combined results of FC ( $\kappa$ light chain restriction, CD19+, CD20+, CD5-, CD10-, and partially $\mathrm{CD} 23+$ ) and immunocytochemistry on the cell block (CD20+, CD3-, bcl1-, bcl2-, bcl6-, and TdT-) with subsequent confirmation by a bone marrow biopsy.

\section{Burkitt Lymphoma}

$\mathrm{BL}$, an aggressive, translocation-associated lymphoma [most commonly of the $t(8 ; 14)$ type, involving the $c$-myc gene], with its middle-sized, rather monomorphic cells, is quite suitable for immunophenotyping with FC. Effusions are common in BL patients and occur in up to $60 \%$ of pleural fluids and $90 \%$ of asctitic fluids in sporadic BL [26]. FC, with its short turnover time, offers a preferable 

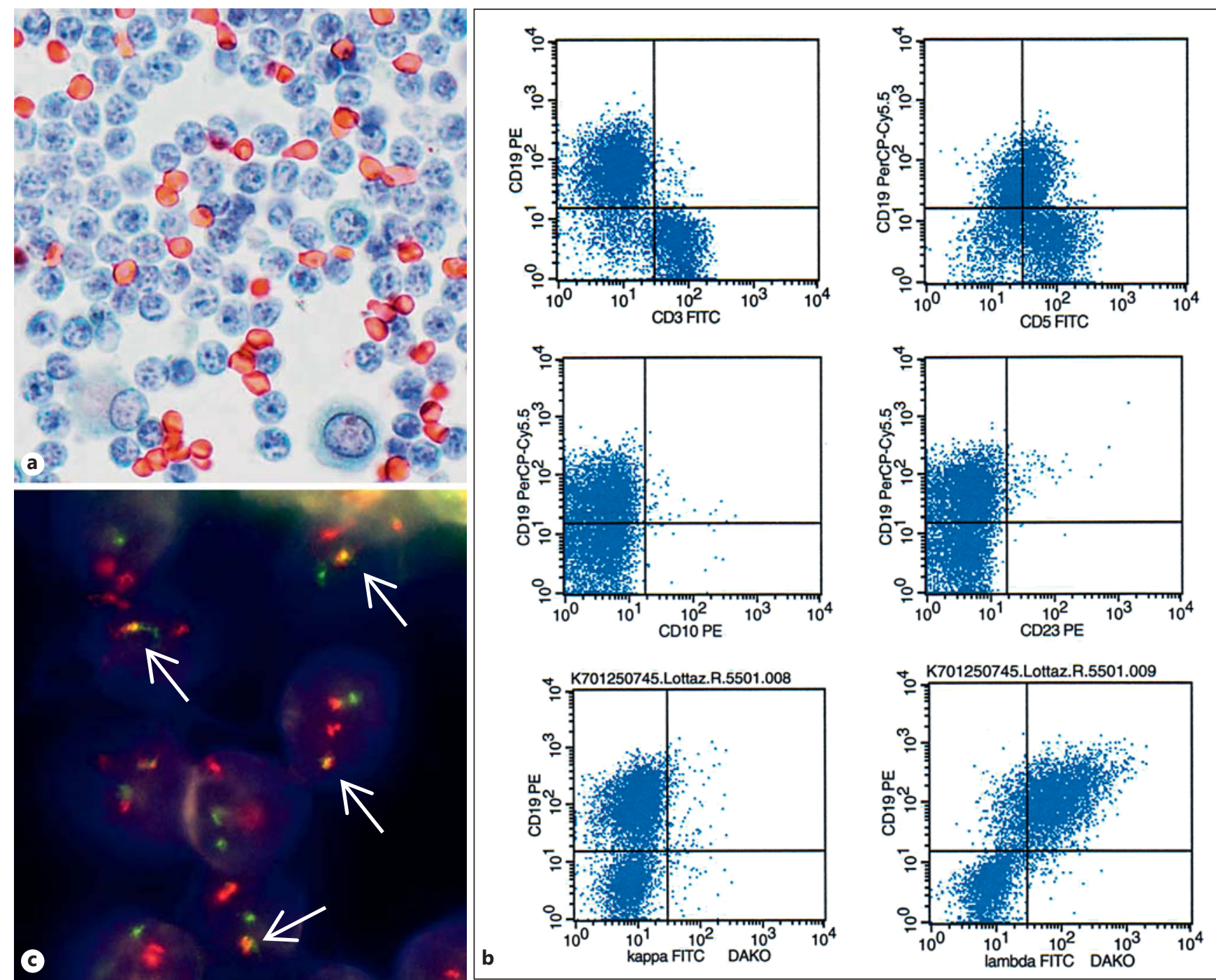

Fig. 3. Ascitic fluid of a 52-year-old male patient with retroperitoneal lymphadenopathy and no history of a tumor. a Sediment smear showing few normal mesothelial cells and numerous small lymphocytes (Papanicolaou, original magnification $\times 400$ ) with a low proliferative activity (MIB1 <5\%; not shown). b FC demonstrating a large CD19-positive B-cell population with $\lambda$ chain re- striction and coexpression of CD5. c FISH analysis with the fusion probe IgH-CCND1 showing fused signals in lymphoma cells (arrows) on sediment smears, indicating the presence of the $t(11 ; 14)$ translocation, diagnostic for Mantle cell lymphoma. Nuclear expression of cyclin D1 was demonstrated immunocytochemically on a cell block (not shown). method for the rapid diagnosis of lymphoma types that may present with dramatic symptoms, such as BL or ALL $[10,26]$. The typical cytomorphology combined with an immunophenotype disclosing a light chain restriction, and $\mathrm{CD} 19+, \mathrm{CD} 20+, \mathrm{CD} 10+$, in the context of a nearly $100 \%$ proliferation index as measured by the Ki67 stain is strongly suggestive of a BL diagnosis and should be confirmed by the presence of rearrangement of the $c-m y c$ gene by further testing (FISH).

\section{Large-Cell Lymphomas}

DLBCL is the most common type of aggressive lymphoma, accounting for approximately $40 \%$ of all lymphoma cases. Serous effusions are a common manifestation of DLBCL. In the study of Chen et al. [2], 18.7\% [41/223, mostly pleural $(n=34)$ or pericardial $(n=5)$ or ascites $(n=7)$, with 5 patients having effusion of 2 body cavities] of patients with this lymphoma type had a cytologically examined serous effusion during the course of their disease, with effusions presented already at the time of the first 


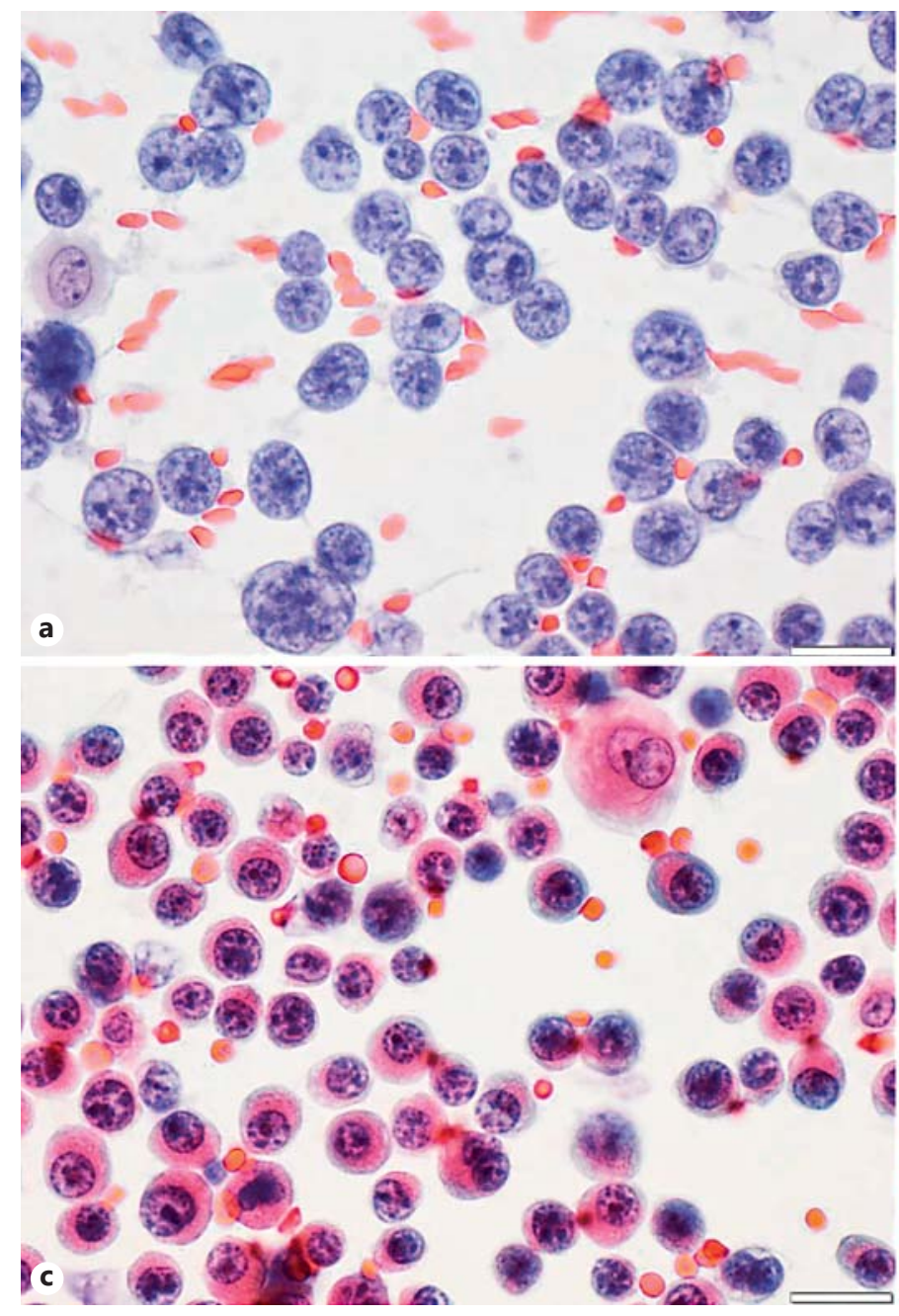

Fig. 4. Examples of lymphatic neoplasia in body fluids, usually not requiring $\mathrm{FC}$, for routine diagnostic purposes. The malignant nature of the cells in such cases is obvious on microscopy, and FC may not be diagnostic due to fragility of the cytoplasm and necrotic debris. a A 67-year-old woman - pleural effusion with cells of a diffuse large B-cell lymphoma. b A 66-year-old woman with

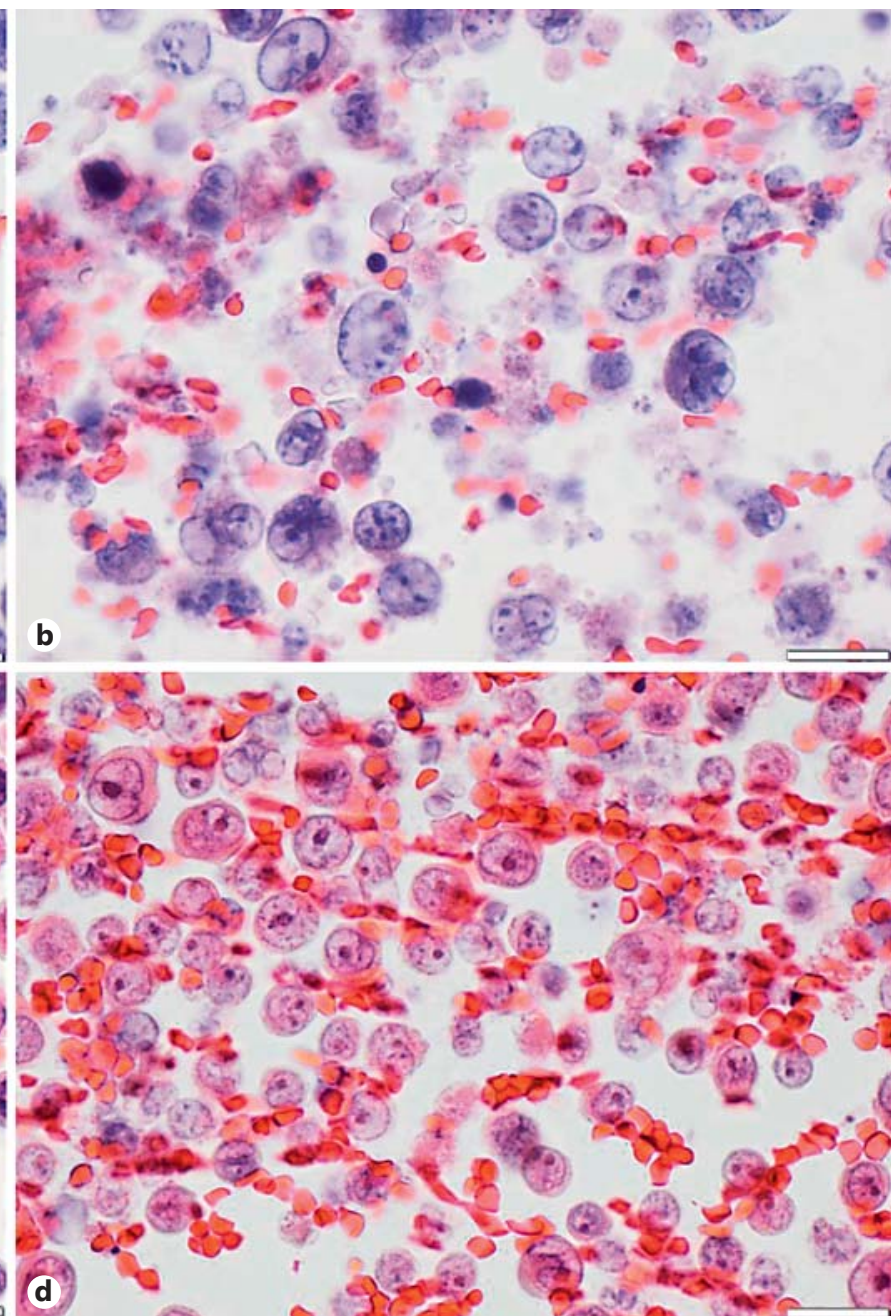

partially necrotic and highly pleomorphic cells of an anaplastic Tcell lymphoma. c A 67-year-old man with isolated mesothelial cells and an otherwise pure population of malignant plasma cells, corresponding to the manifestation of a plasma cell myeloma. d A 37 year-old woman with acute myeloid leukemia. a-d Papanicolaou stain. Original magnification $\times 400$. Scale bars $=20 \mu \mathrm{m}$. diagnosis in $58.5 \%$ of these patients and the rest occurring at 30 days or later. Almost half of these effusions (46\%) were cytologically diagnosed as malignant, containing lymphoma cells. The presence of a malignant effusion in this context was a poor prognostic factor and correlated with and was worst for patients having the effusion at presentation. The diagnosis of malignant cells of DLBCL in the effusion was made via morphologic analysis and it was supplemented in 8 cases by FC, in 2 cases by a clonality analysis, and in 6 cases by cytogenetics. One case of an anaplastic large-cell lymphoma in pleural fluid was studied by FC together with a further 28 histology samples by Kesler et al. [27].

The application of FC to large-cell lymphomas generally carries a substantial risk of false-negative results due to the potential destruction of the fragile cytoplasm of the neoplastic cells with a subsequent artificial loss of surface antigens during cell sorting, with an FC failure rate of $28 \%$ mostly for large-cell lymphoma [9, 12, 27]. Since blastic large-cell lymphomas tend to develop a sometimes extensive necrotic change, FC may not be applicable to such samples (fig. $4 \mathrm{a}, \mathrm{b}$ ). Correlation with conventional micro- 
scopic findings is crucial in such cases. Since malignancy is almost always obvious in these samples on microscopy, the necessary confirmation of the hematologic line of differentiation and the exclusion of carcinomas, melanomas, and sarcomas may be performed immunocytochemically on cell block samples, with FC being less relevant.

\section{T-Cell NHL}

T-cell lymphomas appear to be associated with serous effusions more frequently than B-cell neoplasms do [3]. Precursor T-lymphoblastic lymphoma (T-LBL) comprises approximately $85-90 \%$ of lymphoblastic lymphoma cases, and it is a neoplasm of lymphoblasts committed to the T-cell lineage. This subtype frequently manifests as leukemia and therefore the terminology LBL/ acute lymphoblastic leukemia is usually applied. Das et al. [28] reported pleural effusions in $41.6 \%$ of T-cell lymphoblastic lymphomas as opposed to $3.8 \%$ of cases in other NHL types. A retrospective review [29] also indicated that there is a significantly higher association with pleural effusions in patients with lymphoblastic lymphoma (10/14) than in those with HL (7 of 60). Bhaker et al. [10] studied the spectrum of cytomorphology and FC immunophenotyping in 15 cases of T-LBL, including $10 \mathrm{FNA}$ and 5 effusion (4 pleural and 1 pericardial) samples. FC demonstrated positivity for CD2 (15 of 15 cases), surface CD3 (14 of 15 cases), cytoplasmic CD3 (15 of 15 cases), terminal deoxynucleotidyl transferase (TdT; 8 of 15 cases), CD5 (13 of 15 cases), CD10 (7 of 15 cases), and human leukocyte antigen-D related (HLA-DR; 1 of 15 cases). Dual CD4/CD8 positivity was observed in all cases forming a tight cluster, which is consistent with the cortical T-LBL subtype. In 7 cases TdT (nuclear marker) was negative by FC, while cell block immunocytochemistry was positive in all cases. The combination of cytomorphology and FC enables an accurate and rapid diagnosis of T-LBL on FNA and effusion cytology specimens, thereby obviating the need for a biopsy. FC is extremely useful in these situations because reactive $\mathrm{T}$ cells in effusions demonstrate either CD4 or CD8 positivity but not dual positivity as was observed in the current study. Infiltration by other NHL B-cell types such as CLL/SLL, BL, and MCL also may be considered in the differential diagnosis. The cytomorphology and clinical spectrum are different in these lymphomas; furthermore, FC demonstrates a monoclonal B-cell population.

Peripheral T-cell lymphoma not otherwise specified is a rare, aggressive lymphoma type (25-30\% of all periph- eral $\mathrm{T}$ cell lymphomas), presenting mostly as a generalized lymphadenopathy and rarely affecting extranodal sites (skin and gastrointestinal tract). Its presence in body cavity fluids is rare. A case of peripheral T-cell lymphoma not otherwise specified as a first manifestation in the ascitic fluid of a patient with an HCV infection was described by Ameri et al. [30]. FC of the fluid sample showed an abnormal T-cell population with CD4, CD2, CD5, weak CD3, and an absence of CD7 and TdT expression. Polymerase chain reaction analysis demonstrated clonal $\mathrm{T}$-cell receptor $\gamma$ gene rearrangement, confirming and establishing the diagnosis.

\section{Primary Effusion Lymphoma}

PEL is a uncommon hematologic malignancy presenting in fluids of body cavities without forming tumoral masses in immunocompromised patients, mostly with an HIV infection, and it is caused by superinfection with HHV8 [22, 31]. PEL accounts for about $4 \%$ of all HIVassociated NHL. Morphologically, PEL lymphoma cells exhibit high-grade cytologic features, including an immunoblastic-to-anaplastic appearance, large nuclei with prominent nucleoli, and abundant basophilic cytoplasm. Although there have been some reported cases of T-cell PEL, lymphoma cells of PEL are considered to be generally of B-cell origin. PEL are phenotypically variable, demonstrating a null immunophenotype with evidence of HHV8 infection. PEL show expression of CD45 but lack B-cell and T-cell markers, with a variable presence of activation markers (CD30, CD38, and CD71) and plasma cell differentiation (CD138). As aggressive, blastic lymphomas, they are difficult to characterize by FC and are mostly diagnosed by immunocytochemistry preferably on cell blocks [9].

\section{Hodgkin Lymphoma}

Pleural effusions are common in patients with HL, occurring in $10-30 \%$ of cases $[18,32]$; however, they are less common than in other lymphoma types in children [29]. In the study of Hunter et al. [33], pleural effusions were found radiologically in 26 of 110 patients with HL in various stages of disease, but only 1 patient underwent thoracocentesis with a negative cytologic examination of the fluid sample. Since FC is not the method of choice for the diagnosis of HL, its diagnostic role in this setting is confined to ruling out the differential diagnosis of an NHL. 


\section{Plasma Cell Neoplasms}

Body cavity effusions occurring in the setting of plasma cell myelomas are not common ( $\sim 6 \%$ of cases), and they are nonneoplastic (nonmyelomatous) in most cases due to congestive heart disease or kidney insufficiency, liver dysfunction (cirrhosis, hypoalbuminemia), or inflammation/infection $[18,34-36]$. Rarely (in less than $1 \%$ of cases) a myelomatous infiltration of serous membranes may be the cause of fluid accumulation (fig. 4c). FC with the application of CD19, CD45, CD38, CD138, and CD56 antibodies may be very helpful in diagnosing the presence of plasma cell myeloma cells in the fluid specimen if they are not apparent on microscopy. In contrast to NHL, no expression of CD19 or CD20 is found in plasma cell myelomas. Since neoplastic plasma cells often lack surface light chain expression, alternative methods for the detection of their cytoplasmatic presence (ICC or modified FC protocols) have to be applied in order to avoid false-negative results.

\section{Myeloproliferative Diseases and Myelodysplastic Syndromes}

The presence of a pleural effusion in myelodysplastic syndromes (MDS) is rare and, as in other hemopoietic diseases, it is usually a consequence of infection $[17,18$, 37]. Pleural effusion, secondary to leukemic infiltration, is a rare initial manifestation in patients with MDS. Serous effusions due to disease infiltration in myeloproliferative neoplasms, such as chronic myelocytic leukemia, have been noted in various case reports throughout the literature, mostly in advanced stages, shortly before blastic transformation [18]. The clonal origin of pleural cells in chronic myelocytic leukemia may be identified via conventional cytogenetic methods rather than FC, and they may demonstrate the presence of a Philadelphia chromosome translocation as well as additional chromosomal alterations. The diagnosis of MDS and myeloproliferative neoplasms (acute/chronic myeloid leukemia, essential thrombocythemia, and polycythemia vera, among others) does not typically rely heavily on FC data (fig. 4d). Thus, in patients with a history of or suspicion for these disorders, consultation with a hematopathologist regarding the proper use of FC versus other ancillary studies (cytogenetics and molecular genetics) is the preferred approach. Faiz et al. [37] reported the largest, nonautopsy cohort of patients with acute leukemia and MDS with pleural effusions - 111 patients, 58 (52\%) of whom had a malignant pleural effusion. FC was useful in corroborating the leukemic cause in 20 of the 22 samples submitted for this type of analysis.

\section{Challenges/Pitfalls in the Application of FC to Effusions}

Knowledge of the typical pitfalls of FC in relation to the diagnostics of clinical samples of effusion fluids in the context of exclusion or confirmation of the diagnosis of a lymphoproliferative disease is essential for the proper application of this method. It is important to be familiar with the fact that the lymphocytic population of reactive effusions consists practically exclusively of $\mathrm{T}$ cell lymphocytes and that the large CD3+ population is not indicative of a T-cell lymphoma. On the other hand, there is no specific or definite T-cell FC malignancy marker, and thus in the case of T-cell lymphoma suspicion a molecular T-cell clonality study has to be performed for definite confirmation. In cases of large-cell lymphomas of the $\mathrm{B}$-cell lineage, there is a risk of false-negative diagnoses on FC due to extensive necrosis or mechanical destruction of fragile cytoplasmic borders of the tumor cells, so a correlation with the cytomorphology is obligatory for all cases. There are rare unusual FC patterns, such as unspecific antibody binding $[9,13]$ or loss of surface light chain expression (e.g. in FL), which may require additional studies. The tap-related contamination of body cavity fluids by blood in patients with CLL may lead to the appearance of a small neoplastic cell population in the FC of effusion samples and an apparent falsepositive result for the fluid.

\section{Conclusions}

The application of immunophenotyping by FC to body cavity effusions has proven to be extremely useful for subtyping of lymphocytic populations, both in the context of recurrent known disease and for as yet nondiagnosed lymphomas. The additional use of other auxiliary methods, such as immunocytochemistry and FISH, allowing demonstration of the molecular background of the disease, leads in many cases to the final, clinically relevant definitive specification of the precise type of hematologic malignancy present in the effusions as defined in the current WHO classification. These diagnostic perspectives stress the important role of cytopathologists in the integrative diagnostic process. 


\section{Disclosure Statement}

The author declares no conflict of interests.

\section{References}

1 Gilbert CR, Lee HJ, Skalski JH, Maldonado F, Wahidi M, Choi PJ, Bessich J, Sterman D, Argento $\mathrm{AC}$, Shojaee S, Gorden JA, Wilshire CL, Feller-Kopman D, Ortiz R, Nonyane BA, Yarmus L: The use of indwelling tunneled pleural catheters for recurrent pleural effusions in $\mathrm{pa}$ tients with hematologic malignancies: a multicenter study. Chest 2015;148:752-758.

$\checkmark 2$ Chen Y-P, Huang H-Y, Lin K-P, Medeiros LJ, Chen T-Y, Chang K-C: Malignant effusions correlate with poorer prognosis in patients with diffuse large B-cell lymphoma. Am J Clin Pathol 2015;143:707-715.

-3 Das DK: Serous effusions in malignant lymphomas: a review. Diagn Cytopathol 2006;34: 335-347.

4 WHO Classification of Tumours of Haematopoietic and Lymphoid Tissues, ed 4. Lyon, International Agency for Research on Cancer, 2008.

5 Campo E, Swerdlow S, Harris N, Pileri S, Stein H, Jaffe E: The 2008 WHO classification of lymphoid neoplasms and beyond: evolving concepts and practical applications. Blood 2011 117:5019-5032.

6 Iqbal J, Liu T, Mapow B, Swami VK, Hou JS Importance of flow cytometric analysis of serous effusions in the diagnosis of hematopoietic neoplasms in patients with prior hematopoietic malignancies. Anal Quant Cytol Histo 2010;32:161-165.

7 Laucirica R, Schwartz MR: Clinical utility of flow cytometry in body fluid cytology: to flow or not to flow? That is the question. Diagn Cytopathol 2001;24:305-306.

-8 Czader M, Ali SZ: Flow cytometry as an adjunct to cytomorphologic analysis of serous effusions. Diagn Cytopathol 2003;29:74-78.

-9 Tong LC, Ko HM, Saieg MA, Boerner S, Geddie WR, da Cunha Santos G: Subclassification of lymphoproliferative disorders in serous effusions: a 10-year experience. Cancer Cytopatho 2013;121:261-270.

10 Bhaker P, Das A, Rajwanshi A, Gautam U, Trehan A, Bansal D, Varma N, Srinivasan R: Precursor T-lymphoblastic lymphoma: speedy diagnosis in FNA and effusion cytology by morphology, immunochemistry, and flow cytometry. Cancer Cytopathol 2015;123:557565.

11 Cesana C, Klersy C, Scarpati B, Brando B, Volpato E, Bertani G, Faleri M, Nosari A, Cantoni S, Ferri U, Scampini L, Barba C, Lando G, Morra E, Cairoli R: Flow cytometry versus cytomorphology for the detection of hematologic malignancy in body cavity fluids. Leuk Res 2010 34:1027-1034.

12 Yu GH, Vergara N, Moore EM, King RL: Use of flow cytometry in the diagnosis of lymphoproliferative disorders in fluid specimens. $\mathrm{Di}$ agn Cytopathol 2014;42:664-670.
Mihaescu A, Gebhard S, Chaubert P, Rochat M-C, Braunschweig R, Bosman F, Delacrétaz F, Benhattar J: Application of molecular genetics to the diagnosis of lymphoid-rich effusions: study of 95 cases with concomitant immunophenotyping. Diagn Cytopathol 2002;27:90-95

14 Schmid S, Tinguely M, Cione P, Moch H, Bode B: Flow cytometry as an accurate tool to complement fine needle aspiration cytology in the diagnosis of low grade malignant lymphomas. Cytopathology 2011;22:397-406.

15 Wong JW, Pitlik D, Abdul Karim FW: Cytology of pleural, peritoneal and pericardial fluids in children: a 40-year summary. Acta Cytol 1997; 41:467-473.

16 Shen H, Tang Y, Xu X, Wang L, Wang Q, Xu W, Song H, Zhao Z, Wang J: Rapid detection of neoplastic cells in serous cavity effusions in children with flow cytometry immunophenotyping. Leuk Lymphoma 2012;53:1509-1514.

17 Faiz SA, Sahay S, Jimenez CA: Pleural effusions in acute and chronic leukemia and myelodysplastic syndrome. Curr Opin Pulm Med 2014. 20:340-346

18 Alexandrakis MG, Passam FH, Kyriakou DS, Bouros D: Pleural effusions in hematologic malignancies. Chest 2004;125:1546-1555.

19 Ahmed S, Siddiqui A, Rossoff L, Sison C, Rai K: Pulmonary complications in chronic lymphocytic leukemia. Cancer 2003;98:1912-1917.

20 Sur N, Silverman JF: Synchronous malignancies detected by effusion cytology. Diagn Cytopathol 1998;18:184-187.

21 Vrettos I, Kamposioras K, Peridis S, Aninos D, Kazika S, Spathis A, Karakitsos P, Papadopoulos A: Concurrent pleural infiltration by chronic lymphocytic leukemia and adenocarcinoma of unknown primary site diagnosed by effusion cytology. Diagn Cytopathol 2014;42:151-155.

22 Podder S, Mora M, Patel V, Sivamurthy S: A rare case of bilateral chylothorax: a diagnostic challenge - follicular lymphoma versus primary effusion lymphoma. BMJ Case Rep 2015; 2015.

23 Yonal I, Ciftcibasi A, Gokturk S, Yenerel M, Akyuz F, Karaca C, Demir K, Besisik F, Kalayoglu Besisik S: Massive ascites as the initial manifestation of Mantle cell lymphoma: a challenge for the gastroenterologist. Case Rep Gastroenterol 2012;6:803-809.

24 Keklik M, Yildirim A, Keklik E, Ertan S, Deniz K, Ozturk F, Ileri I, Cerci I, Camlica D, Cetin M, Eser B: Pericardial, pleural and peritoneal involvement in a patient with primary gastric Mantle cell lymphoma. Scott Med J 2015;60: e21-e24.

25 Meykler S, Baloch ZW, Barroeta JE: A case of marginal zone lymphoma with extensive emperipolesis diagnosed on pleural effusion cytology with immunocytochemistry and flow cytometry. Cytopathology 2016;27:70-72.
26 Haddad MG, Silverman JF, Joshi VV, Geisinger KR: Effusion cytology in Burkitt's lymphoma. Diagn Cytopathol 1995;12:3-7.

27 Kesler M, Paranjape G, Asplund S, McKenna R, Jamal S, Kroft S: Anaplastic large cell lymphoma: a flow cytometric analysis of 29 cases. Am J Clin Pathol 2007;128:314-322.

28 Das DK, Gupta SK, Datta U, Sharma SC, Datta BN: Malignant lymphoma of convoluted lymphocytes: diagnosis by fine-needle aspiration cytology and cytochemistry. Diagn Cytopathol 1986;2:307-311.

29 Chaignaud BE, Bonsack TA, Kozakewich HP, Shamberger RC: Pleural effusions in lymphoblastic lymphoma: a diagnostic alternative. J Pediatr Surg 1998;33:1355-1357.

30 Ameri M, Parekh T, Qian Y-W, Elghetany MT, Schnadig V, Nawgiri R: A case of peripheral T-cell lymphoma, not otherwise specified in a HCV and HTLV-II-positive patient, diagnosed by abdominal fluid cytology. J Gastrointest Oncol 2016;7:S96-S99.

31 Wu SI, Hung CC, Chen CH, Tien HF: Primary effusion lymphoma in three patients with chronic hepatitis B infection. J Clin Virol 2009; 44:81-83.

32 Olson PR, Silverman JF, Powers CN: Pleural fluid cytology of Hodgkin's disease: cytomorphologic features and the value of immunohistochemical studies. Diagn Cytopathol 2000;22: 21-24.

33 Hunter B, Dhakal S, Voci S, Goldstein NPN Constine L: Pleural effusions in patients with Hodgkin lymphoma: clinical predictors and associations with outcome. Leuk Lymphoma 2014;55:1822-1826.

34 Keklik M, Sivgin S, Pala C, Eroglu C, Akyol G, Kaynar L, Koker MY, Camlica D, Unal A, Cetin M, Eser B: Flow cytometry method as a diagnostic tool for pleural fluid involvement in a patient with multiple myeloma. Mediterr J Hematol Infect Dis 2012;4:e2012063.

35 Marchesi F, Masi S, Summa V, Gumenyuk S, Merola R, Orlandi G, Cigliana G, Palombi F, Pisani F, Romano A, Spadea A, Papa E, Canfora M, De Bellis F, Conti L, Mengarelli A, Cordone I: Flow cytometry characterization in central nervous system and pleural effusion multiple myeloma infiltration: an Italian national cancer institute experience. $\mathrm{Br} \mathrm{J}$ Haematol 2016;172:980-982.

36 Wang Z, Xia G, Lan L, Liu F, Wang Y, Liu B, Ding Y, Dai L, Zhang Y: Pleural effusion in multiple myeloma. Intern Med 2016;55:339345.

-37 Faiz SA, Bashoura L, Lei X, Sampat KR, Brown TC, Eapen GA, Morice RC, Ferrajoli A, Jimenez CA: Pleural effusions in patients with acute leukemia and myelodysplastic syndrome. Leuk Lymphoma 2013;54:329-335. 